Pacific Journal of Mathematics

HOPE INVARIANTS, LOCALIZATION AND EMBEDDINGS OF 


\title{
HOPF INVARIANTS, LOCALIZATION AND EMBEDDINGS OF POINCARÉ COMPLEXES
}

\author{
Bruce Williams
}

\begin{abstract}
Theorem 0.1. Let $P^{n}$ and $Q^{n}$ be simply connected Poincare complexes such that $P_{(2)} \cong Q_{(2)}$. Assume $n \leqq 2 k-2$. Then $P^{n}$ Poincare embeds in $S^{n+k}$ if and only if $Q^{n}$ Poincare embeds in $S^{n+k}$.
\end{abstract}

The Browder-Sullivan-Casson-Wall embedding theorem [see [23] Chap. 12] then implies the analogous result for manifolds which has also been proven by Rigdon [18] using entirely different methods.

The proof of (0.1) relies upon the following:

TheOREM 0.2. (Localize at odd primes.) Let $X$ be a (q-1)-connected space, and suppose $X \cong \sum \bar{X}$. Then for $m \leqq 3 q-2, \sum^{\infty}$ : $\pi_{m}(X) \rightarrow \pi_{m}^{s}(X)$ has a right inverse.

This result is false if we do not localize at odd primes. For example, Mahowald's $\eta_{j} \in \pi_{2 j}^{s}$ do not desuspend to $\pi_{2 \cdot 2^{j}-3}\left(S^{2^{j}-3}\right)$ (see [14]). The result is also false if $X$ is not a suspension, e.g., $X=S^{i} \times S^{i}$ and $m=2 i$. Since $\pi_{3}^{s}=Z / 24$ and $\pi_{5}\left(S^{2}\right)=Z / 2, m \leqq 3 q-2$ is best possible.

Corollary 0.3. (Localize at odd primes.) Let $X$ be a (q-1)connected space. Then for $i \geqq 1$ and $m \leqq 3 q+2 i-2$.

$\pi_{m+i}\left(\sum^{i} X\right) \cong \pi_{m}^{s}(X) \oplus \pi_{m+i+1}^{s}\left(\sum^{i} X \wedge \sum^{i} X\right)^{z_{2}}$ where $Z_{2}$ acts on $\Sigma^{i} X \wedge \sum^{i} X$ by switching factors. The nonzero elements in the $\pi_{m}^{s}(X)$ term are permanent in the sense that they desuspend to $\Sigma X$ and remain nonzero under the suspension homomorphism. The nonzero elements in the $\pi_{m+i+1}^{s}\left(\Sigma^{i} X \wedge \Sigma^{i} X\right)^{z_{2}}$ term are just flashes in the sense that they do not desuspend and die under a single suspension.

If $X$ is a sphere, then this corollary implies the well known result that for $r \leqq 2 n-2$

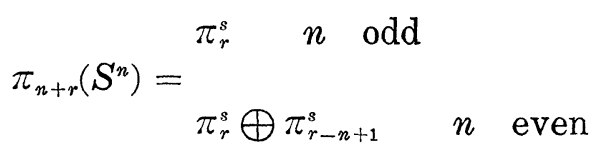

(see [16], [22], [21], and [7] Appendix 2). 
Elsewhere [13] in joint works with Ib Madsen and Larry Taylor (0.2) is applied to the classification of P.L. manifolds.

I.

$$
Q()=\Omega^{\infty} \Sigma^{\infty}() \text {. }
$$

Proof of (0.2). Consider the following commutative diagram

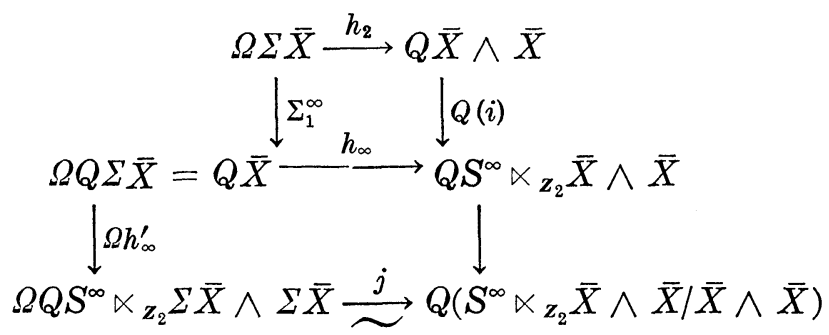

where $h_{2}, h_{\infty}$, and $h_{\infty}^{\prime}$ are Hopf invariant maps coming from stable decompositions of $\Omega \Sigma \bar{X}, Q \bar{X}$, and $Q \Sigma \bar{X}$. (See [15] and [5].) $i: \bar{X} \wedge \bar{X} \rightarrow$ $S^{\infty} \ltimes_{z_{2}} \bar{X} \wedge \bar{X}$ is the inclusion map, and $j$ comes from the homotopy equivalence

$$
\Sigma\left(S^{\infty} \ltimes_{\mathbf{z}_{2}} \bar{X} \wedge \bar{X} / \bar{X} \wedge \bar{X}\right) \rightleftharpoons S^{\infty} \ltimes_{z_{2}} \Sigma \bar{X} \wedge \Sigma \bar{X} \text { (see } 2.3 \text { of [15]) . }
$$

Since $Q$ sends cofibrations to fibrations, the right vertical edge of (1.1) is a fibration sequence. Milgram's EHP sequence (see [15]) implies that $\Omega \Sigma \bar{X}$ is $(3 q-3)$-equivalent to the fibre of $\Omega h_{\infty}^{\prime}$. Since $\Sigma^{\infty}: \pi_{m}(\Sigma \bar{X}) \rightarrow \pi_{m}^{s}(\Sigma \bar{X})$ is induced by $\Sigma_{1}^{\infty}$, we are done if we can show $Q(i)$ has a right inverse when we localize at odd primes.

Consider the following commutative diagram

$$
\begin{aligned}
& \bar{X} \wedge \bar{X} \cong S^{\infty} \times \bar{X} \wedge \bar{X} \\
& S^{\infty} \ltimes_{z_{2}} \bar{X} \wedge \bar{X} \underset{p}{\longleftarrow} S^{\infty} \times_{z_{2}} \bar{X} \wedge \bar{X}
\end{aligned}
$$

where $p$ pinches $S^{\infty} / Z_{2} \times *$ to a point. Notice that $Q(p)_{\text {(odd) }}$ is a homotopy equivalence. Let

$$
t: Q\left(S^{\infty} \times_{z_{2}} \bar{X} \wedge \bar{X}\right) \longrightarrow Q\left(S^{\infty} \times \bar{X} \wedge \bar{X}\right)
$$

be the transfer for the double cover $\pi$. Then $(Q(\pi) \circ t)_{\text {(odd) }}^{-1}$ is a homotopy equivalence, and $t \circ(Q(\pi) \circ t)_{\text {(odd) }}^{-1} \circ Q(p)_{\text {(odd) }}^{-1}$ is a right inverse for $Q(i)_{\text {(odd) }}$.

REMARK. If $\bar{X} \cong \Sigma \overline{\bar{X}}, m \leqq 3 q-4$, and we localize at odd primes; then a right inverse to $\Sigma^{\infty}$ can be derived from the following left 
inverse to Milgram's map $\partial: \pi_{m}\left(S^{\infty} \ltimes_{z_{2}} X \wedge X\right) \rightarrow \pi_{m-1}(X)$ :

$$
\pi_{m-1}(X) \stackrel{H_{X}}{\longrightarrow} \pi_{m}^{s}(X \wedge X)^{Z_{2}} \cong \pi_{m}\left(S^{\infty} \ltimes_{z_{2}} X \wedge X\right) \text {. }
$$

Proof of (0.3). (Localize at odd primes.) By considering diagram (1.1) with $\bar{X}$ replaced by $\Sigma^{i-1} X$, one gets that when $m+i \leqq 3(q+i)-2$

$$
\begin{aligned}
\pi_{m+i}\left(\Sigma^{i} X\right) & \cong \pi_{m+i-1}\left(\Omega \Sigma \Sigma^{i-1} X\right) \\
& \cong \pi_{m+i-1}\left(\Omega Q \Sigma^{i} X\right) \oplus \pi_{m+i}\left(\Omega Q S^{\infty} \ltimes_{z_{2}} \Sigma^{i} X \wedge \Sigma^{i} X\right) \\
& \cong \pi_{m}^{s}(X) \oplus \pi_{m+i+1}^{s}\left(S^{\infty} \ltimes_{z_{2}} \Sigma^{i} X \wedge \Sigma^{i} X\right)
\end{aligned}
$$

where $h_{2}: \pi_{m+i}\left(\Sigma^{i} X\right) \rightarrow \pi_{m+i-1}\left(Q \Sigma^{i-1} X \wedge \Sigma^{i-1} X\right)$ is $1-1$ on $\pi_{m+i+1}^{s}\left(S^{\infty} \ltimes\right.$ $\left.z_{2} \Sigma^{i} X \wedge \Sigma^{i} X\right)$. Thus the nonzero elements in the $\pi_{m+i+1}^{s}\left(S^{\infty} \ltimes_{z_{2}} \Sigma^{i} X \wedge\right.$ $\left.\Sigma^{i} X\right)$ term do not desuspend.

The double cover $\pi: S^{\infty} \times \Sigma^{i} X \wedge \Sigma^{i} X \rightarrow S^{\infty} \times_{z_{2}} \Sigma^{i} X \wedge \Sigma^{i} X$ induces an isomorphism

$$
\pi_{m+i+1}^{s}\left(\Sigma^{i} X \wedge \Sigma^{i} X\right)^{Z_{2}} \cong \pi_{m+i+1}^{s}\left(S^{\infty} \ltimes_{Z_{2}} \Sigma^{i} X \wedge \Sigma^{i} X\right)
$$

Furthermore, the commutativity of the following diagram

$$
\pi_{m: i}\left(\Sigma \Sigma^{i-1} X \wedge \Sigma^{i-1} X\right) \stackrel{\Sigma}{\longrightarrow} \pi_{m+i+1}\left(\Sigma^{i} X \wedge \Sigma^{i} X\right) \longrightarrow \pi_{m+i+1}\left(S^{\infty} \ltimes_{z_{2}} \Sigma^{i} X \wedge \Sigma^{i} X\right)
$$

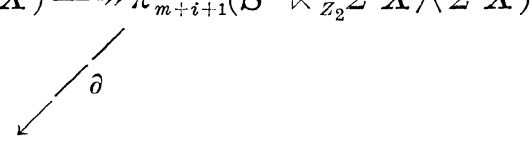

implies that the elements in the $\pi_{m+i+1}\left(S^{\infty} \ltimes_{z_{2}} \Sigma^{i} X \wedge \Sigma^{i} X\right)$-term die after a single suspension.

\section{Open Problems.}

1. Conjecture. If $\alpha \in \pi_{n} Y$ and $\sum^{\infty} a=0$, then $\Sigma^{k} a=0$ for $l_{i} \geqq[n+2 / 2]$.

Surgery theory shows that this conjecture would imply the Hirsh conjecture on embedding $\pi$-manifolds. See [6] for a partial converse when $X=S^{i}$. The Corollary (0.3) implies this conjecture is true when we localize at odd primes.

2. Compute the Hopf invariants of stably trivial elements. If $a \in \pi_{n}(\Sigma X)$ is stably trivial, then in the metastable range $a=\partial(w)$ for some element $w \in \pi_{n+1}\left(S^{\infty} \ltimes_{z_{2}} \Sigma X \wedge \Sigma X\right)$.

Conjecture. $H(a)=t(q(w))$ in $\pi_{n}^{s}(\Sigma X \wedge \Sigma X)$, when $t$ is the transfer of the double cover $S^{\infty} \ltimes \Sigma X \wedge \Sigma X \rightarrow S^{\infty} \times_{z_{2}} \Sigma X \wedge \Sigma X$, and $q$ comes from the stable equivalence

$$
S^{\infty} \times_{z_{2}} \Sigma X \wedge \Sigma X \sim\left(S^{\infty} \times_{z_{2}^{*}}^{*}\right) \vee S^{\infty} \ltimes_{z_{2}} \Sigma X \wedge \Sigma X
$$


The conjecture is equivalent to stably computing the map $t_{1}$ in the cofibre sequence

$$
\Sigma X \wedge X \longrightarrow \Sigma\left(S^{\infty} \ltimes_{z_{2}} X \wedge X\right) \longrightarrow S^{\infty} \ltimes_{z_{2}} \Sigma X \wedge \Sigma X \stackrel{t_{1}}{\longrightarrow} \Sigma X \wedge \Sigma X
$$

3. Conjecture. (Localize at odd primes.) If $m \leqq 3$ (connectivity $X)$, then

$$
\pi_{i}(X) \stackrel{\Sigma^{\infty}}{\longrightarrow} \pi_{i}^{s}(X) \stackrel{\bar{\Delta}}{\longrightarrow} \pi_{i}^{s}(X \wedge X)
$$

is exact, where $\bar{\Delta}$ is the reduced diagonal map.

Since $\pi_{i}^{s}\left(S^{\infty} \ltimes_{z_{2}} X \wedge X\right) \simeq \pi_{i}^{s}(X \wedge X)^{Z_{2}}$, there exists some map $k: \pi_{i}^{s}(X) \rightarrow \pi_{i}^{s}(X \wedge X)$ such that image $\Sigma^{\infty}=$ kernel $k$. Furthermore, an easy Postnikov decomposition argument shows the conjecture is true when localized at 0 .

REMARK. Even if we do not localize, there is a close connection between the Hopf invariant and the reduced diagonal.

If $X \cong \Sigma \bar{X}$, then the pinch map $X \rightarrow X \vee X$ yields a trivialization $\Gamma_{X}:$ cone $X \rightarrow X \wedge X$ of $\bar{\Delta}_{X}: X \rightarrow X \wedge X$.

Proposition. If $f \in[X, Y]$, where $X=\Sigma X$ and $Y=\Sigma \bar{Y}$, then $\Sigma H(f) \in[\Sigma X, Y \wedge Y]$ is represented by

$$
\Sigma X \cong \text { cone } X \cup_{X} \text { cone } X \stackrel{(f \wedge f) \cdot \Gamma_{X} \cup \Gamma_{Y} \cdot c(f)}{\longrightarrow} Y \wedge Y
$$

where $c(f)$ : cone $X \rightarrow$ cone $Y$ is the extension of $f$.

Proof. This is just a reinterpretation of the proof of Theorem 5.14 in [3].

II.

LEMMA 2.1. Let $Z^{n}$ be a simply connected finite $C W$ complex of dimension $n$, and let $\Phi$ be a $S_{\text {(odd) }}^{N}$-fibration over $Z^{n}(N>n+1)$. If $n \leqq 2 q$, then there exists a $S_{\text {(odd) }}^{q-1}$-fibration $\theta^{q}$ over $Z^{n}$ such that $\theta^{q}$ has a cross section, and such that $\theta^{q}$ is stably equivalent to $\Phi$.

Proof. Recall that for simply connected spaces stable $S_{\text {(odd) }}^{N}$ fibrations are classified by $B S G_{\text {(odd) }}$ and $S_{\text {(odd) }}^{q-1}$-fibrations with cross section are classified by $B S F_{q-1 \text { (odd) }}$. (See [20] §4.)

Thus we are done if we can show that the map which classifies $\Phi$ lifts to $B S F_{q-1 \text { (odd) }}$. If $q$ is odd we shall show the map in fact 
lifts to $B S F_{q-2 \text { (odd) }}$. It suffices to show $\pi_{i}\left(S G / S F_{k-1}\right)_{\text {(odd) }}=0$ when $k$ is even and $i \leqq 2 k+1$. Consider the exact sequence:

$$
\begin{aligned}
\pi_{i+k-1}\left(S^{k-1}\right)_{\text {(odd) }} & \stackrel{\Sigma_{1}^{\infty}}{\longrightarrow} \pi_{i \text { (odd) }}^{S} \longrightarrow \pi_{i}\left(S G / S F_{k-1}\right)_{\text {(odd) }} \\
\longrightarrow & \pi_{i+k+2}\left(S^{k-1}\right)_{\text {(odd) }} \stackrel{\Sigma^{\infty}}{\longrightarrow} \pi_{i-1 \text { (odd) }}^{S} .
\end{aligned}
$$

By studying the double suspension (see [7] Appendix 2) one gets that $\Sigma_{1}^{\infty}$ is an epimorphism, $\Sigma^{\infty}$ is an isomorphism, and $\pi_{i}\left(S G / S F_{k-1}\right)_{\text {(odd) }}=0$ when $i \leqq 2 k+1$.

The following result was proved in [10].

THEOREM 2.2. Let $(W, A)^{m}$ be an oriented, finite Poincare pair of formal dimension $m$. Assume $\pi_{1} A \gtrsim \pi_{1} W, m \geqq 6$, and $2 m \geqq 3(n+1)$, where $n=$ homotopy dimension of $W$. Then $(W, A)$ Poincare embeds in $S^{m}$ if and only if $\pi_{m}(W / A)$ contains an element of degree 1.

Although this is a purely homotopy theoretic result, the proof in [10] consists of converting $(W, A)$ to a manifold and then using smooth embedding theory. In §III progress is made towards a homotopy theoretic proof.

Proof of 0.1. Assume $Q$ Poincare embeds in $S^{n+k}$. Let $f: P_{(2)} \rightarrow$ $Q_{(2)}$ be a homotopy equivalence. Let $\eta^{k}$ be the normal fibration for the Poincare embedding of $Q$ in $S^{n+k}$, and let $d \in \pi_{n+k}(T(\eta))$ be the associated normal invariant. $\eta_{(2)}^{k}$ is the $S_{(2)}^{k}$-fibration associated to $\eta$ (see Sullivan [20] for definition and properties). Let $\xi_{t}^{k}=f^{*} \eta_{(2)}^{k} . f^{-1}$ lifts to a map of $S_{(2)}^{k-1}$-fibrations $b\left(f^{-1}\right): S\left(\eta_{(2)}^{k}\right) \rightarrow S\left(\xi_{t}^{k}\right)$ which induces a map of Thom complexes $T\left(f^{-1}\right): T\left(\eta_{(2)}\right) \rightarrow T\left(\xi_{t}\right)$. Notice that $c_{t}=$ $T\left(f^{-1}\right)\left(d_{(2)}\right)$ is a unit in $\pi_{n+k}\left(T\left(\xi_{t}\right)\right)$, i.e. $\operatorname{deg} c_{t} \in z_{(2)}$ is a unit.

Suppose that we could construct a $S^{k-1}$-fibration $\xi$ over $P$ such that $\xi_{(2)}=\xi_{t}$ and a degree 1 map $c: S^{n+k} \rightarrow T(\xi)$. Then $(D(\xi), S(\xi))$ is an oriented, finite Poincare pair of formal dimension $n+k$, and Theorem 2.2 implies there exists a Poincare embedding of $(D(\xi)$, $S(\xi))$ in $S^{n+k}$ which determines a Poincare embedding of $X$ in $S^{n+k}$.

Lemma 2.1 implies there exists a $S_{\text {(odd) }}^{k-1}$-fibration $\xi_{0}$ such that $\xi_{0}$ is stably equivalent to $\gamma_{P \text { (odd) }}$ (where $\gamma_{P}=$ Spivak fibration of $P$ ) and

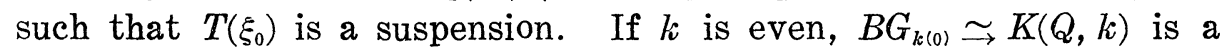
homotopy equivalence where the map is given by the Euler class; and if $k$ is odd, $B G_{k_{(0)}} \cong K\left(Q, 2(k-1)\right.$ ) (see [20] 4.12). Since $\eta^{k}$ is the normal fibration of an embedding in a sphere, the Euler class of $\eta$ and $\xi_{t}$ are trivial. Since $\xi_{0}$ has a cross section, it has trivial Euler class. Thus $\xi_{t}$ and $\xi_{0}$ fit together to yield a $S^{k}$-fibration $\xi^{k}$ 
when $k$ is even. If $k$ is odd, $B G_{k_{(0)}}^{2 k-3} \cong *$, and $\xi_{t}$ and $\xi_{0}$ fit together to yield a $S^{k}$-fibration $\xi^{k}$.

Theorem 0.2 implies that $\pi_{n+k}\left(T\left(\xi^{k}\right)_{\text {(odd) }}\right)$ contains a unit. Furthermore, $\pi_{n+k}\left(T\left(\xi^{k}\right)_{(2)}\right) \cong \pi_{n+k}\left(T\left(\xi_{(2)}\right)\right)$ contains $c_{t}$ which is a unit. Thus $\pi_{n+l k}\left(T\left(\xi^{k}\right)\right)$ contains an element of degree 1 .

III. A Poincare embedding of $(W, A)^{m}$ in $S^{m}$ consists of a finite complex $C$ (the complement) and a map $a: A \rightarrow C$ such that the double mapping cylinder $M(c, a)$ is homotopy equivalent to $S^{m}$, where $c$ is the inclusion of $A$ in $W$. A Poincare embedding determines a deg 1 element $a$ in $\pi_{m}(W / A)$ which is represented by the composition

$$
S^{m} \cong M(c, a) \longrightarrow M(c, a) / C \stackrel{\text { excision }}{\longrightarrow} W / A .
$$

Notice that $\Sigma C \cong(W / A) \bigcup_{a} e^{m+1}$.

In this section we give homotopy theoretic proofs that the hypothesis of Theorem 2.2 imply that

(1) $(W / A) \bigcup_{\alpha} e^{m+1}$ is a suspension

(2) There exists a map $a^{\prime}: \Sigma A \rightarrow(W / A) \bigcup_{\alpha} e^{m+1}$ such that $M\left(\Sigma c, a^{\prime}\right) \cong S^{m+1}$.

If one could prove that the Hopf invariant $H\left(a^{\prime}\right)$ were trivial, then one would have a homotopy theoretic proof of Theorem 2.2.

Browder ([4]) has observed that the composition

$$
\begin{aligned}
b: W \times 0 \cup A \times I \cup W \times 1 & \longrightarrow W \times 0 \cup A \times I \cup W \times 1 / W \times 0 \cong W / A \\
& \longrightarrow W / A \bigcup_{\alpha} e^{m+1}
\end{aligned}
$$

determines an embedding of $(W, A) \times I$ in $S^{m+1}$. In result (2) we are showing Browder's map $b$ factors through

$$
W \times 0 \cup A \times I \cup W \times 1 / W \times 0 \cup W \times 1 \cong \Sigma A .
$$

Proposition 3.1. Let $(W, A)^{m}$ be an oriented, finite Poincare pair of formal dimension $m$. If $\pi_{m}(W / A)$ contains an element $\alpha$ of degree 1 , then the map $j: W \rightarrow W / A$ which pinches $A$ to a point is stably homotopic to a trivial map.

Proof. Let $W^{+}=W \cup\{+\}$ with + as base point. Let $j^{+}=W^{+} \rightarrow$ $W / A$ be the map which sends + to the collapse point and which equals $j$ on $W$. Suppose $e: S^{n} \rightarrow D_{n}\left(W^{+}\right) \wedge W^{+}$is an $n$-duality pairing. Then the map : $\left\{W^{+}, W / A\right\} \rightarrow\left\{S^{n}, D_{n} W^{+} \wedge W / A\right\}$ which sends $f$ to $\left(I_{D_{n} W^{+}} \wedge f\right) \circ e$ is an isomorphism, and we are done if we can show $\left(I_{D_{n} W^{+}} \wedge j^{+}\right) \circ e$ is trivial. 
Let $\bar{J}:(W, A) \rightarrow(W, A) \times W$ be the relative diagonal map. $\bar{J}$ induces a map $\widetilde{\Delta}: W / A \rightarrow W \times W / A \times W \cong W / A \wedge W^{+}$. Since $(W, A)$ satisfies Poincare duality, $e=\widetilde{\Delta} \circ \alpha$ is an $n$-duality map. Notice that the following diagram commutes:

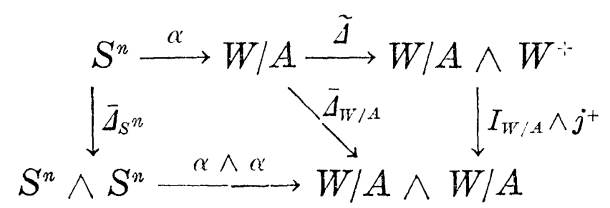

where $\bar{A}_{S^{n}}$ and $\bar{\Delta}_{W / A}$ are reduced diagonal maps. Since $S^{n}$ is a sus-

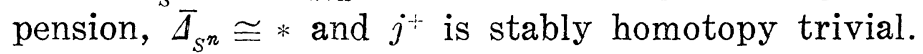

Lemma 3.2. (Jurca [9] Prop. 3.2.) If $3 \leqq q, Z$ is a $(q-1)$-connected $C W$ complex, and $\operatorname{dim} Z \leqq 3 q-3$, then $Z$ desuspends if and only if $\bar{\Delta}_{Z} \cong *$.

Proof of (1). Poincare duality implies $W / A$ is $(m-n-1)$-connected. $\bar{\Delta}_{W / A}=\left(I_{W / A} \wedge j^{+}\right) \circ \widetilde{\Delta}$ which is stably trivial by Proposition 3.1. Since $m=\operatorname{dim} W / A \leqq 2$ (connectivity $W / A \wedge W / A)=2(2(m-n)$ $-1), \bar{\Delta}_{W / A}$ is in fact unstably trivial and Lemma 3.3 implies $W / A$ is a suspension. Then $W / A \bigcup_{\alpha} e^{m+1} \cong(W / A)^{m-1}$ is also a suspension.

Proof of (3). Consider the cofibration sequence $A \stackrel{c}{\rightarrow} W \stackrel{j}{\rightarrow} W / A \stackrel{l}{\rightarrow}$ $\Sigma A$. Since $j$ is homotopy trivial, $l$ has a left inverse $l^{\prime}$. Let $a^{\prime}$ be the composition $\Sigma A \stackrel{l^{\prime}}{\rightarrow} W / A \rightarrow W / A \bigcup_{\alpha} e^{n+1}$. An easy homology and van Kampen's argument shows, $M\left(\Sigma c, a^{\prime}\right) \cong S^{m+1}$.

\section{REFERENCES}

1. W. D. Barcus, The stable suspension of an Eilenberg-MacLane space, Trans. Amer. Math. Soc., 96 (1960), 101-114.

2. J. C. Becker and D. H. Gottlieb, The transfer map and fiber bundles, Topology, 14 (1975), 1-12.

3. J. M. Boardman and B. Steer, On Hopf invariants, Comment. Math. Helv., 42 (1967), 180-221.

4. W. Browder, Embedding smooth manifolds, in Proc. I.C.M. (Moscow, 1966), Mir, (1968), 712-719.

5. F. R. Cohen, J. P. May, and L. R. Taylor, Splittings of certain spaces CX, to appear, Proc. Cambridge Phil. Soc.

6. H. Glover and G. Mislin, Metastable annihilation in the homotopy groups of spheres, Notices of the Amer. Math. Soc., 18 (1971), 385.

7. D. Husemoller, Fibre Bundles, 2nd ed., Springer-Verlag, Graduate Texts in Mathematics 20.

8. L. Jones, Path spaces: A geometric representation for Poincare spaces, Ann. of Math., 97 (1973), 306-343.

9. D. R. Jurca, Obstructions to desuspending a complex, Northwestern Thesis, 1973.

10. L. L. Larmore and B. Williams, Single obstructions to embedding and Boardman- 
Vogt little cubes, to appear.

11. J. Levine, A classification of differentiable knots, Ann. of Math., 82 (1965), 15-50.

12. J. Levine, On differentiable embeddings of simply connected manifolds, Bull. Amer. Math. Soc., 69 (1963), 806-809.

13. I. Madsen, L. Taylor and B. Williams, Tangential homotopy equivalence, to appear.

14. M. Mahowald, A new infinite family in ${ }_{2} \pi^{s_{*}}$, Topology, 16 (1977), 249-256.

15. J. Milgram, Unstable Homotopy From the Stable Point of View, Lecture Notes No. 368, Springer-Verlag, 1974.

16. J. Moore, The double suspension and p-primary components of the homotopy groups of spheres, Bol. Soc. Mat. Mexicana (2), 1 (1956), 28-37.

17. F. Quinn, Surgery on Poincaré and normal spaces, Bull. Amer. Math. Soc., 78 (1972), 262-267.

18. R. Rigdon, P-equivalences and embeddings of manifolds, Proc. London Math. Soc.,

11 (1975), 233-244.

19. F. Rouch, Transfer in Generalized Cohomology Theories, Princeton thesis, 1971.

20 D. Sullivan, Geometric Topology. Part I. Localization, Periodicity, and Galois Symmetry, Notes, M.I.I., 1970.

21. S. Thomeier, Über eine Beziehurg zwischen unstabilen und stabilen Homotopiegruppen von Sphären, Arch. Math., 15 (1964), 351-353.

22. H. Toda, On the double suspension $E^{2}$, J. Inst. Polytech. Osaka City University Ser. A., 7 (1956), 103-145.

23. C. T. C. Wall, Surgery on Compact Manifolds, Academic Press, 1971.

Received July 15, 1978. The author was partially supported by an NSF grant.

University of Notre Dame

NOTRE DAME, IN 46556 


\section{PACIFIC JOURNAL OF MATHEMATICS}

\section{EDITORS}

DONALD BABBITT (Managing Editor)

University of California

Los Angeles, California 90024

Hugo RossI

University of Utah

Salt Lake City, UT 84112

C. C. MOORE and ANDrew OGG

University of California

Berkeley, CA 94720
J. DUgundjI

Department of Mathematics University of Southern California Los Angeles, California 90007

R. FinN aNd J. Milgram Stanford University Stanford, California 94305

\section{ASSOCIATE EDITORS}

E. F. BECKENBACH

B. H. NeUMANN

F. WOLF

K. YosHIDA

\section{SUPPORTING INSTITUTIONS}

UNIVERSITY OF BRITISH COLUMBIA CALIFORNIA INSTITUTE OF TECHNOLOGY UNIVERSITY OF CALIFORNIA MONTANA STATE UNIVERSITY UNIVERSITY OF NEVADA, RENO NEW MEXICO STATE UNIVERSITY OREGON STATE UNIVERSITY UNIVERSITY OF OREGON
UNIVERSITY OF SOUTHERN CALIFORNIA STANFORD UNIVERSITY UNIVERSITY OF HAWAII UNIVERSITY OF TOKYO UNIVERSITY OF UTAH WASHINGTON STATE UNIVERSITY UNIVERSITY OF WASHINGTON 


\section{Pacific Journal of Mathematics}

\section{Vol. 84 , No. 1 \\ May, 1979}

Michael James Beeson, Goodman's theorem and beyond ...............

Robert S. Cahn and Michael E. Taylor, Asymptotic behavior of multiplicities

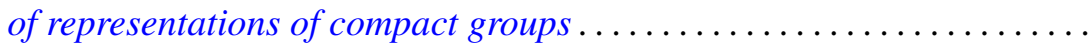

Douglas Michael Campbell and Vikramaditya Singh, Valence properties of

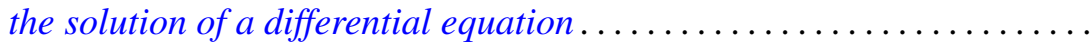

J.-F. Colombeau, Reinhold Meise and Bernard Perrot, A density result in spaces of Silva holomorphic mappings .....................

Marcel Erné, On the relativization of chain topologies .................

Le Baron O. Ferguson, Uniform and $L_{p}$ approximation for generalized

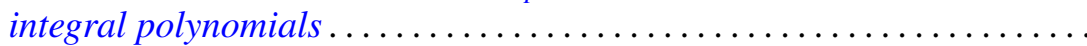

Kenneth R. Goodearl and David E. Handelman, Homogenization of regular rings of bounded index..................................

Friedrich Haslinger, A dual relationship between generalized

Abel-Gončarov bases and certain Pincherle bases .................

Miriam Hausman, Generalization of a theorem of Landau. .

Makoto Hayashi, 2-factorization in finite groups

Robert Marcus, Stochastic diffusion on an unbounded domain ........... 143

Isabel Dotti de Miatello, Extension of actions on Stiefel manifolds....

C. David (Carl) Minda, The hyperbolic metric and coverings of Riemann surfaces...

Somashekhar Amrith Naimpally and Mohan Lal Tikoo, On $T_{1}$-compactifications.

Chia-Ven Pao, Asymptotic stability and nonexistence of global solution for a semilinear parabolic equation ....

Shigeo Segawa, Harmonic majoration of quasibounded type ...

Sze-Kai Tsui and Steve Wright, The splitting of operator algebras ...

Bruce Williams, Hopf invariants, localization and embeddings of Poincaré complexes....

Leslie Wilson, Nonopenness of the set of Thom-Boardman maps ...

Alicia B. Winslow, There are $2^{\mathrm{c}}$ nonhomeomorphic continua in $\beta R^{n}-R^{n}$ 